\title{
The Ion-Binding Characteristics of Reconstituted Collagen
}

\author{
By A. WEINSTOCK,* P. C. KING AND R. E. WUTHIER \\ Harvard School of Dental Medicine and Forsyth Dental Center, Boston, Mass. 02115, U.S.A.
}

(Received 23 June 1966)

\begin{abstract}
The ion-binding capacity of highly purified reconstituted calf-skin collagen, and the effects of these ions on the precipitation and solubility of the collagen, were studied with a variety of salt solutions at ionic strength $0 \cdot 16$ and $\mathrm{pH} 7 \cdot 4$. Only a small percentage of the total theoretically available anionic and cationic groups was available for ion-binding. In view of this, it appears that most of the ionizable groups of collagen are involved in intramolecular or intermolecular linkages, or both. Nevertheless, marked differences in the binding of the various ions by collagen were observed. Bivalent cations were bound in extremely small but remarkably similar quantities. In contrast, sodium was bound both in much higher and more variable quantities. Of the anions, pyrophosphate and sulphate were bound in the largest quantities, followed by phosphate, fluoride and chloride, in that order. Despite the minimal uptake by collagen of bivalent cations, they prevented the aggregation of tropocollagen into fibrils, and disaggregated fibrillar collagen. In the presence of multivalent anions, tropocollagen aggregated readily and its fibrillar stability was maintained. On the basis of the imbalance in the binding of ion pairs by the sodium pyrophosphate- and sodium phosphate-treated collagens, it was apparent that a reduced number of side-chain carboxyl groups were dissociated in the presence of these salts.
\end{abstract}

Considerable work has been done with collagen, both from the standpoint of its unique structure and chemical properties (Bear, 1952; Gross \& Kirk, 1958; Gustavson, 1956; Harrington \& Hippel, 1961; Hodge \& Schmitt, 1961) and its relation to biological mineralization (Fitton-Jackson, 1957; Glimcher, 1960; Taves \& Neuman, 1963). Many of its biological functions are probably dependent on its large content of polar amino acids (Eastoe, 1955). The fact that collagen is the major structural element of bone matrix and appears to be intimately associated with bone mineral led us to investigate its ion-binding properties. This paper deals with the ion-binding characteristics of a highly purified reconstituted calf-skin collagen and with the effect of these ions on its structural and physical properties.

By measuring the binding capacity of collagen for various ions, it was possible to: (1) compare the effective availability of positively or negatively charged groups with their theoretical availability; (2) establish whether or not collagen preferentially binds certain ionic species; (3) observe the effects of the individual ions on the protein.

* Present address: Department of Anatomy, McGill University, Montreal, Canada.

\section{MATERIALS AND METHODS}

Preparation of dialysis tubing. Dialysis tubing (cellulose casing; Union Carbide Corp. Food Products Division, Chicago, Ill., U.S.A.) was boiled in three successive changes of $5 \%(w / v) \mathrm{NaHCO}_{3}$ and rinsed exhaustively with distilled and finally deionized water, to remove all traces of salt.

\section{Preparation of purified reconstituted collagen by repeated precipitation from acetic acid}

Cleaning and grinding of hide. Foetal (Holstein) calf skin was obtained immediately after slaughter. Fat and flesh were removed at $0-5^{\circ}$. The skin was washed in $0.9 \%$ $\mathrm{NaCl}$, shaved, cut into strips 1 in. $\times$ in. and frozen on solid $\mathrm{CO}_{2}$ powder. The frozen strips were ground in a Wiley mill with solid $\mathrm{CO}_{2}$ chips.

Removal of soluble impurities. The ground hide was soaked overnight with shaking at $0-5^{\circ}$ in $0.5 \mathrm{M}$-sodium acetate. The extraction was repeated, the residue collected on cheese-cloth and the extracts discarded.

Extraction of collagen. The residue was suspended with shaking at $0-5^{\circ}$ in $0.2 \mathrm{M}$-sodium citrate buffer at $\mathrm{pH} 4.5$ overnight. The extract was filtered through cheese cloth and retained. The extraction was repeated three times and the extracts pooled.

Purification of collagen. The extract was clarified by high-speed centrifugation $(10000-15000 \mathrm{~g})$ at $0-5^{\circ}$ followed 
by pressure filtration $(0.45 \mu$ pore size; Millipore Filter Corp., Bedford, Mass., U.S.A.). The filtrate was dialysed against $1 \%(\mathrm{w} / \mathrm{v}) \mathrm{NaCl}$ buffered with $0.02 \mathrm{M}$-tris at pH 7.6. The non-diffusible material was then precipitated by warming to $37^{\circ}$, the precipitate collected by centrifugation at $1250 \mathrm{~g}$, and the supernatant discarded. The heatprecipitated material was dissolved in cold $0.05 \mathrm{~N}$-acetic ncid, the solution filtered by pressure filtration and the insoluble residue discarded. The clear viscous filtrate was rlialysed at $0-5^{\circ}$ against $1 \%(w / v) ~ N a C l$ buffered with (1.02 $\mathrm{M}$-tris at $\mathrm{pH} 7 \cdot 6$. The resulting precipitate was collosted by centrifugation (10000-15000g) and the supernatant discarded. The precipitate was redissolved in $0.05 \mathrm{~N}$-acetic acid and the solution clarified by pressure filtration. Reprecipitation in the above manner was repeated five times, with pressure filtration between each precipitation. The final precipitate was left in dialysis bags for salt treatment.

\section{Preparation of citrate buffer extract of collagen (Gallop, 1955)}

The method described for preparing reconstituted collagen was employed up to and including the clarification step. The clear viscous extract was dialysed against $0.02 \mathrm{M}-\mathrm{Na}_{2} \mathrm{HPO}_{4}$. The precipitates were collected by centrifugation $(1250 \mathrm{~g})$, redissolved in citrate buffer and the solution clarified by pressure filtration. Reprecipitation was repeated five times, with pressure filtration between steps, and the final product was redissolved in citrate buffer for use as tropocollagen.

\section{Chemical analysis}

For cation analysis, the collagens were digested with $70 \%(\mathrm{w} / \mathrm{v}) \mathrm{HClO}_{4}$ and diluted to the appropriate concentrations for analysis. For the determination of fluoride, chloride and sulphate, the collagens were treated in centrifuge tubes for $30 \mathrm{~min}$. at room temperature with $\mathrm{N}-\mathrm{HCl}$, $\mathrm{N}-\mathrm{H}_{2} \mathrm{SO}_{4}$ and $\mathrm{N}-\mathrm{HCl}$ respectively, to liberate bound ions. After centrifugation $(1250 \mathrm{~g})$ of the collagens, appropriate samples of the supernatants were used for analysis. Phosphate and pyrophosphate were analysed as total phosphate after digestion of the collagens for $30 \mathrm{~min}$. with $70 \%$ $\mathrm{HClO}_{4}$ at $180^{\circ}$ and with $6 \mathrm{~N}-\mathrm{HCl}$ at $105^{\circ}$ respectively. For the orthophosphate determination, $10 \mathrm{mg}$. samples of collagen were digested in $0.5 \mathrm{ml}$. volumes of $70 \% \mathrm{HClO}_{4}$, whereas pyrophosphate was determined after hydrolysing $10 \mathrm{mg}$. samples of collagen in $1 \mathrm{ml}$. volumes of $6 \mathrm{~N}-\mathrm{HCl}$. Calcium, strontium, magnesium and sodium were analysed directly with a Perkin-Elmer atomic absorption spectrophotometer, sodium samples being handled only in poly. propylene test tubes. Fluoride, chloride, phosphate and sulphate were determined colorimetrically by the methods of Wharton (1962), Conway (1962), Martin \& Doty (1949) and Stoffyn \& Keane (1964) respectively. For hydroxyproline analysis, the collagen was hydrolysed for $16 \mathrm{hr}$. in $6 \mathrm{~N}-\mathrm{HCl}$ at $105^{\circ}$ and the hydroxyproline determined by the method of Neuman \& Logan (1950).

\section{Experimental procedure}

Expt. 1: treatment of the purified precipitated collagen with various salt solutions. The purified precipitated collagen was further dialysed against several changes of $1 \%(w / v)$ $\mathrm{NaCl}$, maintained at neutral $\mathrm{pH}$ with AG-1 (X8; OHform) anion-exchange resin (California Corp. for Biochemical Research, Los Angeles, Calif., U.S.A.) to remove the tris buffer. The collagen was then dialysed to exhaustion against $0.16 I$ salt solutions, maintained at $\mathrm{pH7.4} \pm 0.2$ with appropriate AG-50W (X8; $\mathrm{H}^{+}$form) or AG-1 (X8; $\mathrm{OH}^{-}$form) ion-exchange resin.

The treated collagens were then exhaustively dialysed against deionized water, maintained at neutral $\mathrm{pH}$ with anion-exchange resin ( $\mathrm{OH}-$ form), to remove unbound ions. The washed salt-treated collagen was subsequently freezedried and analysed for various ions.

Expt. 2: heat precipitation of soluble collagen. After the purified precipitated collagen was exhaustively dialysed against the various $0 \cdot 16 I$ salt solutions, the non-diffusible residue was centrifuged at $1250 \mathrm{~g}$. Portions of the supernatant of each sample, containing tropocollagen that was not precipitated as fibrils at $0 \cdot 16 I$, as well as any collagen fibrils solubilized by the salt treatment, were warmed to $37^{\circ}$ to test for heat precipitation.

Expt. 3: treatment of the citrate buffer extract of collagen with various salt solutions. The citrate buffer extract of collagen was similarly treated with the same salts, except that it was used in solution (tropocollagen) rather than as precipitated fibrils. These experiments were performed to study the effect of the various ions on the precipitation of collagen from solution. Chemical analyses were not done.

\section{RESULTS}

Table 1 illustrates the effect of the various electrolytes on the reconstituted collagen. Whereas $\mathrm{Ca}^{2+}, \mathrm{Sr}^{2+}$ and $\mathrm{Mg}^{2+}$ tended to inhibit fibril formation (Expt. 3), previously reconstituted fibrils were solubilized by these ions and their $640 \AA$ periodicity was lost when they were examined with the electron microscope (Expt. 1). The collagen fibrils were relatively unaffected by the presence of the other electrolytes. In Expt. 2, collagen remaining soluble after dialysis against the various $0 \cdot 16 I$ salt solutions yielded a precipitate when warmed to $37^{\circ}$ in each sample except those containing calcium, strontium or magnesium salts.

Table 2 summarizes the quantitative ion-binding by collagen of the various salts used. With two exceptions, only a small quantity of the total theoretically available functional groups did, in fact, bind ions. Although $\mathrm{Sr}^{2+}, \mathrm{Ca}^{2+}$ and $\mathrm{Mg}^{2+}$ were bound in extremely small quantities, they appeared to have a great effect on the structural properties of the collagen. The binding of phosphate by collagen $(97 \mu \mathrm{moles} / \mathrm{g}$.) was essentially the same as that reported by Glimcher \& Krane (1964). Compared with phosphate, however, there were $276 \mu$ moles of pyrophosphate and $152 \mu$ moles of sulphate bound/g. of collagen. The amount of fluoride bound was $60 \%$ that of phosphate and almost four times the amount of chloride bound. This fluoride-binding was almost five times that found with a phosphate-precipitated collagen simi- 
Table 1. Effects of electrolytes on reconstituted collagen

All salt solutions were at $I 0 \cdot 16$ and $\mathrm{pH} 7 \cdot 4 \pm 0 \cdot 2$. The collagen fibrils used in Expts. 1 and 2 were prepared from an acetic acid extract; the tropocollagen used in Expt. 3 was in citrate buffer. In Expt. 1, the purified collagen fibrils were dialysed against the various salt solutions. In Expt. 2, the collagen that remained soluble after dialysis against the salt solutions was warmed to $37^{\circ}$ to test for heat precipitation. In Expt. 3, the tropocollagen was dialysed against the various salt solutions.

$$
\begin{aligned}
& \text { Expt. } 1 \text { : effect of } \\
& \text { electrolytes on } \\
& \text { collagen fibrils } \\
& \text { on dialysis }
\end{aligned}
$$

\section{Salt}

Sodium chloride

Sodium fluoride

Sodium phosphate

Sodium pyrophosphate

Sodium sulphate

Calcium chloride

Strontium chloride

Magnesium chloride

\begin{abstract}
Dissolution
\end{abstract}

-
-
$=$
$\overline{+}$
+
+

Expt. 2: heat precipitation of soluble collagen

Precipitation
Expt. 3: effect of electrolytes on tropocollagen on dialysis

Precipitation

Table 2. Ion-binding in reconstituted collagen

\begin{tabular}{|c|c|c|}
\hline Salt & $\begin{array}{c}\text { Cation } \\
\text { ( } \mu \text { equiv./g. of collagen) }\end{array}$ & $\begin{array}{c}\text { Anion } \\
\text { ( } \mu \text { equiv./g. of collagen) }\end{array}$ \\
\hline Sodium chloride & $11 \cdot 7 \pm 1 \cdot 6$ & $14 \cdot 8 \pm 1 \cdot 0$ \\
\hline Sodium fluoride & $14 \cdot 3 \pm 1 \cdot 5$ & $57 \cdot 8 \pm 2 \cdot 4$ \\
\hline Sodium phosphate & $18 \cdot 0 \pm 2 \cdot 9$ & $96 \cdot 9 \pm 3 \cdot 6$ \\
\hline Sodium pyrophosphate & $615 \cdot 0 \pm 5 \cdot 8$ & $275 \cdot 8 \pm 9 \cdot 8$ \\
\hline Sodium sulphate & $129 \cdot 2 \pm 6 \cdot 1$ & $152 \cdot 3 \pm 5 \cdot 2$ \\
\hline Calcium chloride & $0.63 \pm 0.05$ & $8 \cdot 4 \pm 1 \cdot 0$ \\
\hline Strontium chloride & $0.50 \pm 0.01$ & $10 \cdot 8 \pm 1 \cdot 1$ \\
\hline Magnesium chloride & $1.06 \pm 0.05$ & $11 \cdot 8 \pm 1 \cdot 6$ \\
\hline
\end{tabular}

The collagen fibrils were prepared from an acetic acid extract. The salt solutions were $I 0.16$ and the $\mathrm{pH}$ was adjusted and maintained at $\mathrm{pH} 7 \cdot 4 \pm 0.2$ with appropriate ion-exchange resins in either the $\mathrm{H}^{+}$or $\mathrm{OH}^{-}$form. Values represent the means \pm S.I.M. of at least quadruplicate determinations.

larly treated with fluoride (A. Weinstock, unpublished work). Gross \& Kirk (1958) have shown that F- acts as an accelerating agent for collagen fibrillogenesis in vitro.

\section{DISCUSSION}

These studies indicated that there were striking differences in the binding of ions by collagen under identical experimental conditions. The total theoretically available anionic and cationic groups in ox-bone collagen (Eastoe, 1955) at $\mathrm{pH} \mathrm{7.4,} \mathrm{after}$ correction for amide nitrogen and for the dissociation of histidine (pK 7.25; Kenchington \& Ward, 1954), are 898 and $901 \mu \mathrm{moles} / \mathrm{g}$. of collagen respectively. This indicates that at this $\mathrm{pH}$ there are approximately equal amounts of free anionic and cationic groups/g. of collagen. Tropocollagen in 0.1 M-tris buffer has an isoelectric point at $\mathrm{pH} \mathrm{8.3}$ (Bensusan \& Hoyt, 1958); however, the isoionic point of gelatin is at approx. pH 9.4 (Eastoe, Long \& Willan, 1961), suggesting that a positive net charge exists at neutral $\mathrm{pH}$.

Only a small percentage of the total theoretically available anionic groups were, in fact, free to bind cations. Exceptions to this were the pyrophosphateand sulphate-treated collagens, which showed a high $\mathrm{Na}^{+}$uptake. Under physiological conditions, therefore, most of the anionic groups in collagen are probably involved in internal linkages.

Of all the ions studied, bivalent cations were bound least. $\mathrm{Ca}^{2+}, \mathrm{Sr}^{2+}$ and $\mathrm{Mg}^{2+}$, in a decreasing order of potency, dissolved the collagen fibrils, and after prolonged dialysis yielded a clear viscous gel. In addition, in the presence of these metal ions, tropocollagen could not be precipitated into native-type fibrils (Table 2, Expt. 3). It is conceivable that bivalent cations might form electrovalent bonds between key carboxyl groups and thereby prevent the $640 \AA$ periodic aggregation of 
the macromolecules. Davison \& Drake (1966) suggest the presence of specifically located carboxyl groups that enhance fibrogenesis, and microelectrophoretic studies (Gilbert, 1960) support the concept of $\mathrm{Ca}^{2+}$ binding to carboxyl moieties in collagen. In the present study the extremely low uptake of these ions and their marked inhibition of fibril formation suggested that this binding must have been highly specific. That heat precipitation failed to occur when samples of tropocollagen containing $\mathrm{Ca}^{2+}, \mathrm{Sr}^{2+}$ or $\mathrm{Mg}^{2+}$ were warmed to $37^{\circ}$ (Table 2, Expt. 2) could be explained as above, or on the basis that bivalent cations might have denatured the collagen. It is evident from these observations and from the findings of others (Gustavson, 1956; Gallop, Seifter \& Meilman, 1957) that bivalent cations have a significant effect on collagen, both in the tropocollagen and fibrillar phases.

Of the anions investigated, pyrophosphate and sulphate were bound by collagen in the highest quantities. The large binding of pyrophosphate by collagen is significant in view of the suggestion by Cartier \& Lanzetta (1961) that certain of the $\epsilon$-amino groups of bone collagen may be bonded to pyrophosphate and involved in apatite nucleation. Studies by Wuthier, Grøn \& Irving (1964) confirmed the presence of a fraction of 6 -amino groups in bone collagen that was apparently strongly associated with the bone mineral and would not react with 1-fluoro-2,4-dinitrobenzene except in the terminal stages of demineralization. Sulphate-binding was noteworthy in the light of the electrophoretic studies of Bensusan \& Hoyt (1958) that have shown that tropocollagen binds large quantities of sulphate. Wood (1960) has also demonstrated that collagen fibril formation was accelerated in the presence of chondroitin sulphates $A$ and $C$ and keratosulphate.

The relatively large quantities of phosphate bound by collagen agreed with the findings of Glimcher \& Krane (1964). The possible significance of phosphate-binding to collagen in relationship to calcification was discussed by these authors (Glimcher \& Krane, 1962, 1964). It was observed that collagen was precipitated much more rapidly and completely from solutions in phosphate buffer than in any of the other salt solutions. Moreover, it was apparent that collagen was precipitated more readily and exhibited greater stability in the presence of salts with multivalent anions. This was in direct contrast with the effect of bivalent cations, which tended to disaggregate collagen fibrils (Table 1). In previous studies it was observed that more phosphate was bound at $\mathrm{pH} 4.0$ than at neutral $\mathrm{pH}$. This would be expected, since there would theoretically be more charged basic groups at low $\mathrm{pH}$ as well as a substantial decrease in the number of charged carboxyl groups. Martin, Mergenhagen \& Scott (1961) in fact demonstrated that there were more titratable imidazole groups in unbanded fibrils at low $\mathrm{pH}$ than in $640 \AA$-banded fibrils at pH 7.4. It is not unreasonable to assume that phosphate could bind at these strategic sites, as well as to the cationic groups of lysine, hydroxylysine and arginine. Kuhn, Grassmann \& Hofmann (1958) have shown that phosphate is bound to the guanidine groups of arginine in $640 \AA-$ banded fibres. Further, when a solution of tropocollagen was dialysed against phosphate at $\mathrm{pH} 4 \cdot 0$, it could not be reconstituted into fibrils of the native type when redialysed against phosphate buffer at neutral pH (A. Weinstock, unpublished work). This finding may indicate that at low $\mathrm{pH}$ firm phosphate bonds were created at strategic cationic sites which prevented $640 \AA$ native-type fibril formation. Hallsworth (1964) determined that approx. $61 \%$ of the total amino groups in collagen were available to react with 1-fluoro-2,4dinitrobenzene; however, he forewarned against relating the reactivity of $\epsilon$-amino groups with fluorodinitrobenzene to the binding of phosphate by the same groups. Our findings tend to justify his caution.

Great care was taken during these experiments to avoid possible error due to fluctuations in $\mathrm{pH}$. Considering the distinct differences in the ionbinding patterns of the various ions studied, the effect of trace amounts of $\mathrm{H}^{+}$and $\mathrm{HCO}_{3}-$ ions present in the systems was probably insignificant. If collagen is isoionic at $\mathbf{p H ~} \mathbf{9 . 4}$ as is gelatin (Eastoe et al. 1961), small quantities of anions would probably be present in the dialysis bag as counterions during dialysis at $\mathrm{pH} \mathrm{7.4} \mathrm{owing} \mathrm{to} \mathrm{the} \mathrm{Donnan}$ effect; however, in view of the exhaustive dialysis against deionized water maintained as close as possible to neutral $\mathrm{pH}$, these effects should not have been a significant factor in the measurement of ion-binding by the collagen. This was evident from the apparent discrepancy in the balance of electrical charges seen especially in the pyrophosphate- and orthophosphate-treated collagens.

For example, with the data obtained from the sodium pyrophosphate-treated collagen, the following calculations can be made: if the $\mathrm{p} K_{4}$ and $\mathrm{p} K_{3}$ of pyrophosphate at infinite dilution are taken to be 8.22 and 5.77 respectively, after correction for ionic strength $\left(\mathrm{p} K^{\prime}=\mathrm{p} K-0.5 \sqrt{I}\right)$ the $\mathrm{p} K$ values at $I 0.16$ would be 8.02 and 5.57 respectively. Since $276 \mu$ moles of pyrophosphate/g. of collagen were bound at $\mathrm{pH} 7 \cdot 4$ (Table 2 ), it can be calculated from the Henderson-Hasselbalch equation that $882 \mu$ equiv. of cationic groups/g. of collagen were bound to pyrophosphate, leaving $19 \mu$ equiv. of cationic groups unbound. Conversely, $615 \mu$ equiv. of $\mathrm{Na}^{+} / \mathrm{g}$. of collagen were bound 
(Table 2), leaving $283 \mu$ equiv. of anionic groups unbound. It is thus apparent that $264 \mu$ equiv. of anionic groups/g. of collagen were unbalanced by either counter-ions or cationic groups in the collagen at $\mathrm{pH} 7 \cdot 4$. However, if it is assumed that the $\mathrm{pH}$ of the solution had not been properly controlled and that the $p K$ values were unaffected, the $\mathrm{pH}$ required to obtain balance between electrical charges in the sodium pyrophosphate-treated collagen can be calculated. Since this would require a large decrease in $\mathrm{pH}$, all of the imidazole groups of histidine (pK 7.25; Kenchington \& Ward, 1954) in collagen would presumably be in the associated form. This would result in a total of $898 \mu$ equiv. of negative and $937 \mu$ equiv. of positive charges/g. of collagen. Since $283 \mu$ equiv. of negative charges/g. of collagen would be left unbalanced after $\mathrm{Na}+$-binding, to maintain electrical balance there would have to be an equal amount of positive charges/g. of collagen left unbalanced after pyrophosphate-binding. Thus $654 \mu$ equiv. of positive charges/g. of collagen would have to be bound to pyrophosphate. In view of the fact that $276 \mu$ moles of pyrophosphate/g. of collagen were bound, the net charge/pyrophosphate ion would have to have been $2 \cdot 37$. Since $\mathrm{H}_{2} \mathrm{P}_{2} \mathrm{O}_{7}{ }^{2-}$ $+\mathrm{HP}_{2} \mathrm{O}_{7}{ }^{3-}$ must add up to $276 \mu$ equiv. and $2 \times \mathrm{H}_{2} \mathrm{P}_{2} \mathrm{O}_{7}{ }^{2-}+3 \times \mathrm{HP}_{2} \mathrm{O}_{7}{ }^{3-}$ must equal $654 \mu$ equiv., the amount of each can be calculated to be 174 and $102 \mu$ equiv./g. of collagen respectively. From the Henderson-Hasselbalch equation, the pH can thus be calculated to be 5.34, a value far below $\mathbf{7 \cdot 4}$.

Although the $\mathrm{pH}$ of the deionized water may have decreased to values somewhat less than $7 \cdot 4$ during the extended dialysis, the fact that other salt-treated collagens did not display this discrepancy in ion-binding suggests that the effect was due to the salts and not to change in $\mathrm{pH}$. That a change in the apparent dissociation constants of the anionic and cationic groups could occur was even more plausible in view of electron-microscope studies, which have shown that the charged groups are not randomly distributed, but are localized in specific areas along the collagen triple-helix (Bear, 1952 ; Kuhn, Grassmann \& Hofmann, 1957; Hodge \& Schmitt, 1961). Physicochemical studies on other proteins have shown that distinct effects on the dissociation constants of side-chain groups can be caused by local environment (Alberty, 1953). In fact, Bensusan \& Hoyt (1958), Veis, Anesey \& Cohen (1958) and Davison \& Drake (1966) have shown that tropocollagen exhibits marked changes in its isoelectric and isoionic points in the presence of different salt solutions, indicating that variable binding of ions by the tropocollagen had occurred.

Whether ions are bound directly by electrovalent bonding (Bello, 1963), or exert an influence in- directly by interacting with low-entropy structurally-bound water (Hippel \& Wong, 1963a,b), is uncertain. Rubin et al. (1965) and Davison \& Drake (1966) presented evidence indicating that peptides protrude from the triple-helix body of native tropocollagen. They postulated that these 'telopeptides' may participate in the biological control of fibrillogenesis through alterations of intra- and inter-molecular bonding. In view of the fact that these peptides contain appreciable amounts of polar amino acids (Drake, Davison, Bump \& Schmitt, 1966), it is possible that they may play a role in the binding of ions by collagen; nevertheless, this remains to be studied.

This work was supported with funds supplied by U.S. Public Health Service Grant no. DE-00876, National Institute for Dental Research. A.W. was a Research Fellow in Periodontology, Harvard School of Dental Medicine, and a Dental Research Fellow, National Research Council of Canada at the time this work was done. R.E.W. is a Career Development Awardee, U.S. Department of Health, Education and Welfare. The encouragement of Dr J. T. Irving is gratefully acknowledged.

\section{REFERENCES}

Alberty, R. A. (1953). In The Proteins, vol. 1, part A, p. 461. Ed. by Neurath, H. \& Bailey, K. New York: Academic Press Inc.

Bear, R. S. (1952). Advanc. Protein Chem. 7, 69.

Bello, J. (1963). Biochemistry, 2, 276.

Bensusan, H. B. \& Hoyt, B. L. (1958). J. Amer. chem. Soc. $80,719$.

Cartier, P. \& Lanzetta, A. (1961). Bull. Soc. Chim. biol., Paris, 43, 91.

Conway, E. J. (1962). Microdiffusion Analysis and Volumetric Error, 5th ed., p. 301. London: Crosby Lockwood and Sons.

Davison, P. F. \& Drake, M. P. (1966). Biochemistry, 5, 313.

Drake, M. P., Davison, P. F., Bump, S. \& Schmitt, F. O. (1966). Biochemistry, 5, 301.

Eastoe, J. E. (1955). Biochem. J. 61, 589.

Eastoe, J. E., Long, J. E. \& Willan, A. L. D. (1961). Biochem. J. 78, 51.

Fitton-Jackson, S. (1957). Proc. Roy. Soc. B, 146, 270.

Gallop, P. M. (1955). Arch. Biochem. Biophys. 54, 486.

Gallop, P. M., Seifter, S. \& Meilman, E. (1957). J. biophys. biochem. Cytol. 8, 545.

Gilbert, I. G. F. (1960). Biochim. biophys. Acta, 40, 156. Glimcher, M. J. (1960). In Symposium on Calcification in Biological Systems, p. 421. Ed. by Sognnaes, R. F. Washington: American Association for the Advancement of Science.

Glimcher, M. J. \& Krane, S. M. (1962). In Radioisotopes and Bone, p. 393. Ed. by Lacroix, P. \& Budy, A. M. Oxford: Blackwell Scientific Publications Ltd.

Glimcher, M. J. \& Krane, S. M. (1964). Biochemistry, 8, 195.

Gross, J. \& Kirk, D. (1958). J. biol. Chem. 238, 355.

Gustavson, K. H. (1956). The Chemistry and Reactivity of Collagen, p. 173. New York: Academic Press Inc.

Hallsworth, A. S. (1964). Biochem. J. 93, 255. 
Harrington, W. F. \& Hippel, P. H. von (1961). Advanc. Protein Chem. 16, 1.

Hippel, P. H. von \& Wong, K. Y. (1963a). Biochemistry, 2, 1387.

Hippel, P. H. von \& Wong, K. Y. (1963b). Biochemistry, 2, 1399.

Hodge, A. J. \& Schmitt, F. O. (1961). In Macromolecular Complexes, p. 19. Ed. by Edds, M. V., jun. New York: The Ronald Press Co.

Kenchington, A. W. \& Ward, A. D. (1954). Biochem. J. 58, 202.

Kuhn, K., Grassmann, W. \& Hofmann, U. (1957). Naturwissenschaften, 44, 538.

Kuhn, K., Grassmann, W. \& Hofmann, U. (1958). Z. Naturf. 18, 154.

Martin, G. R., Mergenhagen, S. E. \& Scott, D. B. (1961). Biochim. biophys. Acta, 49, 245.
Martin, J. B. \& Doty, D. M. (1949). Analyt. Chem. 21, 965. Neuman, R. E. \& Logan, M. A. (1950). J. biol. Chem. $184,299$.

Rubin, A. L., Drake, M. P., Davison, P. F., Pfahl, D., Speakman, P. T. \& Schmitt, F. O. (1965). Biochemistry, 4, 181.

Stoffyn, P. \& Keane, W. (1964). Analyt. Chem. 36, 397.

Taves, D. R. \& Neuman, W. F. (1963). Factors Controlling Calcification, p. 50. Rochester: University of Rochester, Atomic Energy Project.

Veis, A., Anesey, J. \& Cohen, J. (1958). In Recent Advances in Gelatin and Glue Research, p. 155. Ed. by Stainsby, G. London: Pergamon Press Ltd.

Wharton, H. W. (1962). Analyt. Chem. 84, 1296.

Wood, G. C. (1960). Biochem. J. 75, 605.

Wuthier, R. E., Grøn, P. \& Irving, J. T. (1964). Biochem. J. 92, 205. 